\title{
On hyponormal operators and related classes of operators
}

\author{
ARIYADASA ALUTHGE
}

\author{
Date of Receiving : $\quad 01.04 .2017$ \\ Date of Revision : 15.06 .2017 \\ Date of Acceptance : 15.08 .2017
}

\begin{abstract}
There are many classes of operators defined based on operator inequalities. The class of hyponormal operators is perhaps the first such class of operators. An operator $T$ defined on a Hilbert space $H$ is called hyponormal if $T^{*} T \geq T T^{*}$, where $T^{*}$ is the adjoint of the operator $T$. Since then, many other classes of operators have been introduced, mainly by weakening or modifying the inequality $T^{*} T \geq T T^{*}$ in various ways. Among these classes of operators are semihyponormal operators, $p$-hyponormal operators (for $0<p<1 / 2$ ), log-hyponormal operators, and $w$-hyponormal operators. Hyponormal operators possess many interesting properties. And the other classes of operators possess the same properties or somewhat weaker properties. In this paper, we will give a review of these classes of operators and their properties.
\end{abstract}

\section{Introduction}

Let $H$ be a separable Hilbert Space and let $L(H)$ denote the algebra of bounded linear operators on $H$. The adjoint operator $T^{*}$ of an operator $T$ on $H$ is defined by $\left\langle T^{*} x, y\right\rangle=\langle x, T y\rangle$ for all $x$ and $y$ in $H$. An operator $T \in L(H)$ is called self-adjoint if $T^{*}=T$ or equivalently if $\langle T x, x\rangle$ is real for all $x \in H$. A self-adjoint operator $T \in L(H)$ is called positive semi-definite, denoted $T \geq 0$, if $\langle T x, x\rangle \geq 0$ for all $x \in H$. An operator $T \in L(H)$ is called normal if $T^{*} T=T T^{*}$ (or equivalently $\left\|T^{*} x\right\|=\|T x\|$ for all $x \in H$ ) and hyponormal if $T^{*} T \geq T T^{*}$ (or equivalently $\left\|T^{*} x\right\| \leq\|T x\|$ for all $x \in H)$. An operator $U \in L(H)$ is called unitary if $U^{*} U=I=U U^{*}$, where $I$ is the identity operator on $H$, an isometry if $U^{*} U=I$ or equivalently, $\|U x\|=\|x\|$ for all $x \in H$, and a partial isometry if $U^{*} U$ is projection from $H$ to a subspace of $H$. The polar decomposition of an operator $T \in L(H)$ is denoted by $T=U|T|$, where $|T|=\left(T^{*} T\right)^{\frac{1}{2}}$ and $U$ is a partial isometry from $\overline{R(|T|)}$ to $\overline{R(T)}$ such that $N(U)=N(|T|)$ with $\overline{N()}$ and $\overline{R()}$ being the closures of null space and the range, respectively, of an operator. The spectrum of an operator $T$ is defined by $\sigma(T)=\left\{z \in \mathbb{C}:(T-z I)^{-1}\right.$ does not exist $\}$ and the spectral radius defined by $r_{s p}=\sup \{|z|: z \in \sigma(T)\}$.

2010 Mathematics Subject Classification. 47A12, 47A63, 47B15, 47B20.

Key words and phrases. Hyponormal operators, p-Hyponormal operators.

Communicated by: Takeaki Yamazaki 